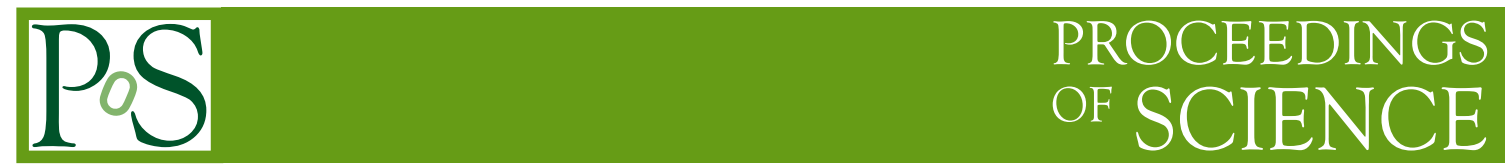

\title{
RD42: Radiation hard diamond sensors
}

\author{
Gregor Kasieczka for the RD42 Collaboration \\ ETH Zurich \\ E-mail: Gregor.Kasieczka@cern.ch
}

The RD42 Collaboration at CERN is investigating chemical vapor deposition diamond as a material for tracking detectors operating in extreme radiation environments. We present an overview of the latest developments from RD42. The status of diamond based luminosity monitors for the upcoming CERN Large Hadron Collider (LHC) run is described followed by a discussion of recent beam test measurements of the pulse height dependence on the incoming charged particle flux for single-crystal and poly-crystalline diamond sensors. Finally the use of 3D geometries for CVD diamond sensors is reviewed.

24th International Workshop on Vertex Detectors

1-5 June 2015

Santa Fe, New Mexico, USA 


\section{Introduction}

The next generation of experiments in particle physics will probe new regimes in energy and luminosity. These two conditions imply that measurements will have to be carried out in areas of very high radiation. Chemical Vapor Deposition (CVD) diamond is a radiation tolerant alternative to silicon sensors for precision tracking detectors. Basic material properties of diamond, such as large displacement energy and band gap are the main factors influencing its radiation hardness. Further features are low leakage current, low dielectric constant and fast signal collection and the ability to operate at room temperature.

During the past few years many CVD diamond devices have been manufactured and tested. The RD42 collaboration [12] has measured the signal response of single-crystal and poly-crystalline CVD diamond detectors irradiated to particle fluences up to $1.8 \cdot 10^{16}$ protons $/ \mathrm{cm}^{2}$ [4]. As a consequence of these measurements, diamond pixel tracking detectors have been implemented as beam condition and luminosity monitors for both general purpose LHC experiments - Compact Muon Solenoid (CMS) and the A Toroidal LHC ApparatuS (ATLAS) - whose radiation environments require the use of radiation tolerant sensor materials.

\section{Diamond detector applications}

The ATLAS Diamond Beam Monitor (DBM) upgrade [6] has been installed and is collecting data in the ongoing 2015 LHC run. This device is populated with poly-crystalline CVD diamond sensors with an active area of $18 \times 21 \mathrm{~mm}^{2}$.

Previously the CMS experiment had employed diamond pixel sensors in the pilot run of the Pixel Luminosity Telescope (PLT) operating in 2012-2013 [1]. For this single-crystal CVD diamonds with a smaller area of $4.5 \times 4.5 \mathrm{~mm}^{2}$ were used. The sensors experienced a particle flux of more than $4 \mathrm{MHz} / \mathrm{cm}^{2}$ during the PLT pilot run. The total integrated fluence during the entire run was estimated by a FLUKA $[2,5]$ calculation to be $\sim 5 \times 10^{13}$ neutral hadrons $/ \mathrm{cm}^{2}$ and $\sim 5 \times 10^{13}$ charged hadrons $/ \mathrm{cm}^{2}[8]$.

\section{Pixel and PAD results}

After receiving a relatively low fluence of $\sim 1 \times 10^{13}$ hadrons $/ \mathrm{cm}^{2}$ a pulse height dependence on particle flux with the pulse height decreasing with increasing particle flux was observed for the PLT sensors [11]. This drop in pulse height was stronger than the dependence predicted from previous RD42 beam test data motivating a systematic study whose first results — initially presented in [13] — are summarised here.

Poly-crystalline and single-crystal CVD diamond sensors were irradiated to a neutron fluence of $5.0 \pm 0.5 \times 10^{13} \mathrm{n} / \mathrm{cm}^{2}-$ similar to the total fluence observed by the PLT during the pilot run in CMS. These sensors, together with a non-irradiated single-crystal sample and single-crystal samples irradiated during the PLT pilot run, were measured in a beam of $250 \mathrm{MeV} / \mathrm{c}$ pions. The pion flux could be controlled and was varied between $1 \mathrm{kHz} / \mathrm{cm}^{2}$ and $20 \mathrm{MHz} / \mathrm{cm}^{2}$. To test the impact of electrostatic effects and the weighting field on the charge collection two different detector geometries were tested: a pad and a pixel geometry. All samples were $\approx 500 \mu \mathrm{m}$ thick and pixel detectors were produced with an electrode size of $75 \mu \mathrm{m} \times 125 \mu \mathrm{m}$ and a pitch of $100 \mu \mathrm{m} \times 150 \mu \mathrm{m}$. 
The same telescope - accommodating up to six pixel planes and with silicon pixel planes used as the front and back plane - was used test detectors with pixel and pad geometries. When measuring pixel detectors the middle four planes were occupied by the CVD diamond pixel sensors under test. When measuring pad detectors these planes were removed and the pad detector box was placed in that position. An image of the telescope in pad configuration can be seen in Figure 1. For both configurations a coincidence of the so-called fast-OR signal [7] from front and back plane was required as trigger.

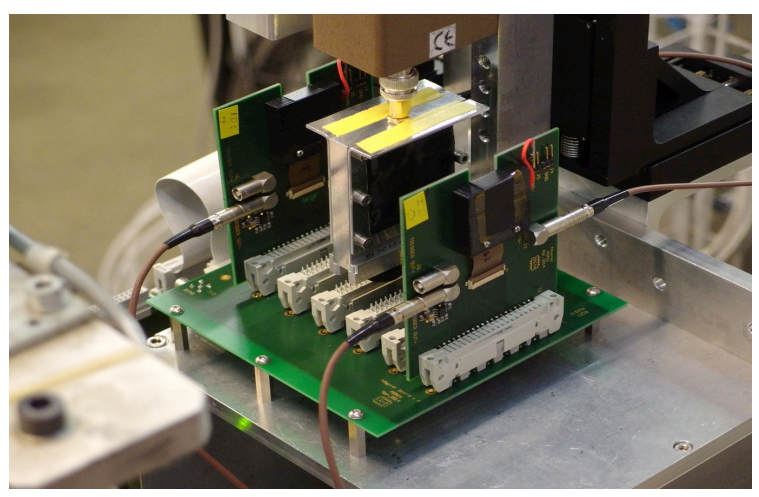

Figure 1: The telescope shown in the pad arrangement. First and last planes are CMS silicon pixel detectors used for triggering. The pad detector can be removed and four additional pixel planes can be inserted to convert it to the pixel only arrangement [13].

Pixel detectors were read out with a standard CMS PSI46v2 pixel readout chip (ROC) [7] and a PSI46 test board. For the pad detector an Ortec 142A preamplifier [9] was used in combination with an Ortec 450 amplifier for amplification and pulse shaping. Finally the waveforms from the pad detector were digitised using a DRS4 evaluation board [10].

To extract the pulse height from the pad detector waveformsan integration window of $70 \mathrm{~ns}$ around the signal peak was chosen and the detector pedestal level was subtracted. The position of the signal peak was assumed to be constant during each run. Finally, for each detector the pulse height distribution was re-scaled with a common factor so that the mean pulse height for the lowest flux run was one.

The change of mean pulse height with incident particle flux is presented in Figure 2. Unirradiated single-crystal as well as neutron irradiated poly-crystalline samples show no pulse-height dependence on flux while a drop of $\approx 10 \%$ is observed for single-crystal samples after neutron irradiation or irradiation in the PLT pilot run.

For the analysis of the pixel detector results the ADC values from the pixel readout system were first converted to the collected charge using an internal calibration procedure. Adjacent pixels that exceeded the threshold charge were added to form a cluster and the charge of the cluster calculated as the sum of the pixels' charges. For the analysis of the pulse height five of the six tracking planes were designated as reference planes and the remaining plane as the plane under test. Exactly one cluster was required in each of the reference planes and a straight track was fit to these clusters. No requirement was made on the presence of a hit in the plane under test. If the constructed track fulfilled basic quality requirements its intersection point with the plane under test 

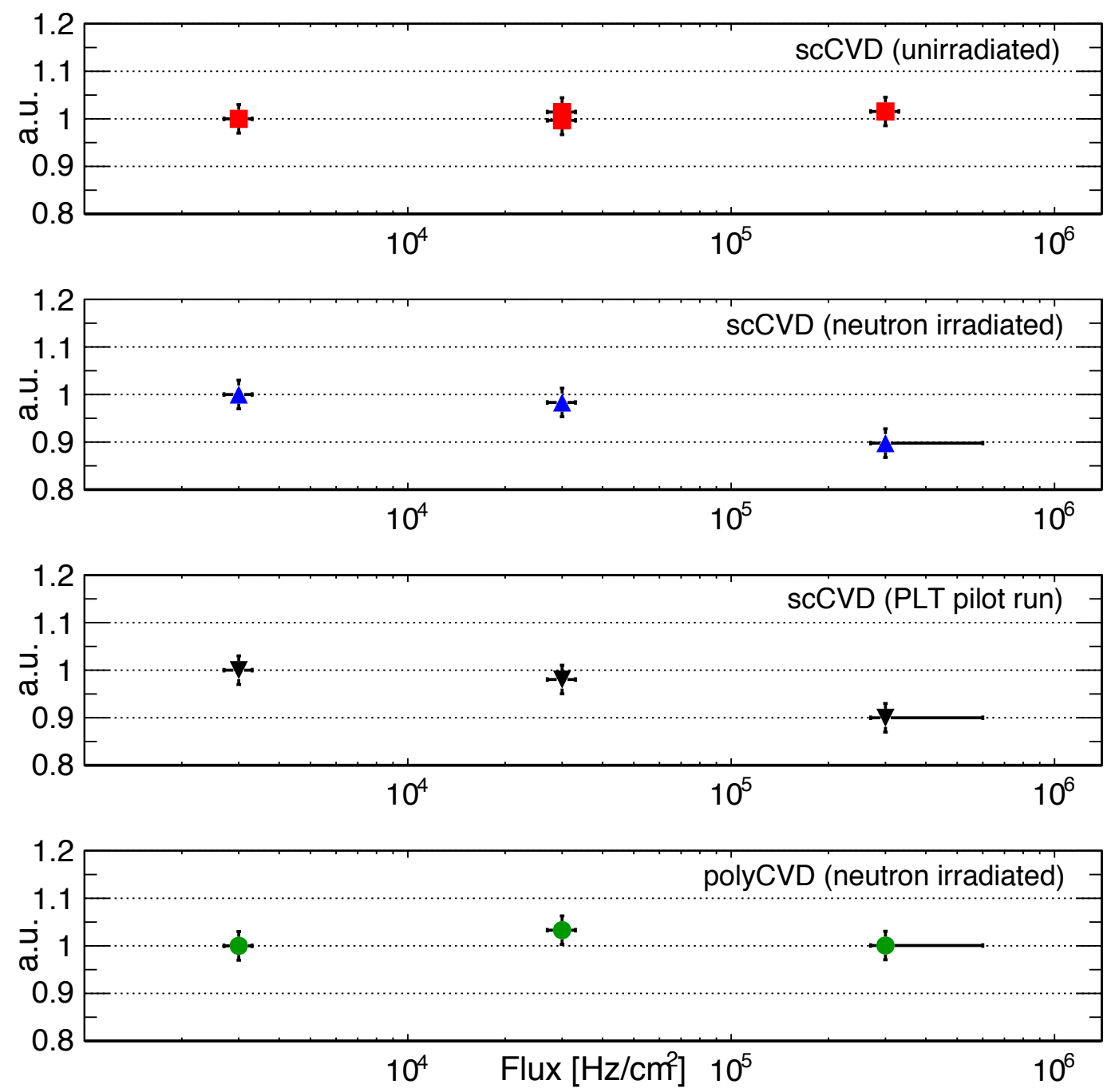

Figure 2: The mean values of the pulse height distributions for all pad geometry detectors versus the incident particle flux. The statistical uncertainty on the points is approximately $1 \%$. We estimate a $3 \%$ systematic uncertainty by comparing several procedures used to estimate the mean [13].

was calculated and the pulse height of the plane under test calculated as the sum of charges of a single cluster in a four pixel radius around the intersection point. The pulse heights for each device under test were then re-scaled with a device specific scale factor so that the most probable value of the pulse height distribution at the lowest flux was one. The systematic uncertainty on the average pulse height was estimated to be $\approx 0.4 \%$ using a silicon sensor.

The average pulse height as function the incident particle flux for a single-crystal CVD diamond sensor is shown in Figure 3 (top left). A small decrease of 5\% between lowest and highest flux can be observed. This is attributed to a combination of charge sharing between pixels and the per-pixel threshold of $\approx 3000 \mathrm{e}$. As can be seen in Figure 3 (top right) the mean cluster size increases with increasing flux, reducing the charge collected by an individual pixel. The pulse height 
of the single-crystal sample irradiated during the PLT pilot run is shown in Figure 3 (bottom). Here the pulse height is reduced by a factor of two when going from the lowest to the highest flux. This is consistent with the observation made in the PLT pilot run [11] but much larger than the flux dependence observed with pad readout electronics. This implies that the pixel readout threshold plays a role in the large decrease in pulse height observed for the irradiated single-crystal pixel sensor.
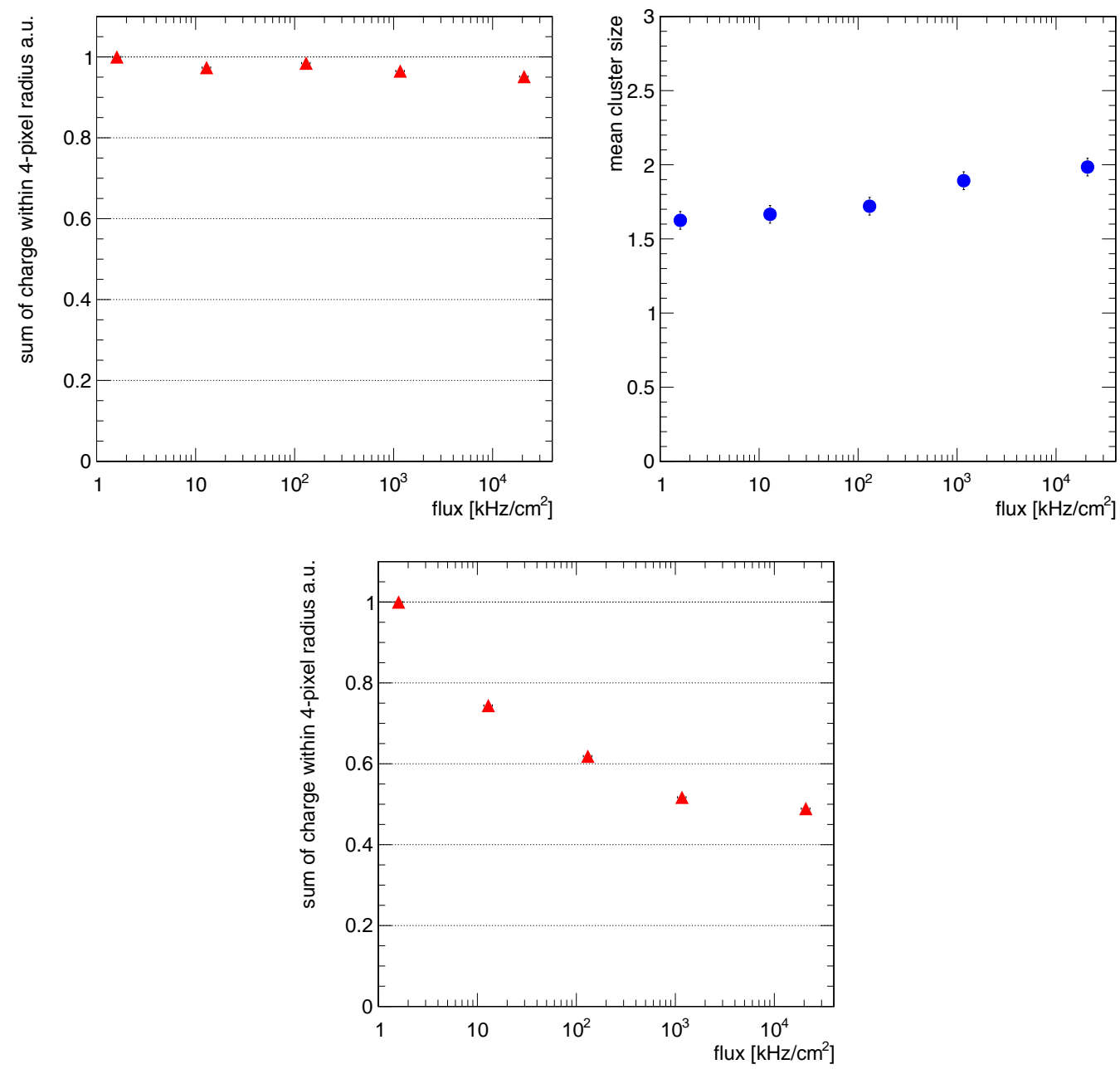

Figure 3: The average pulse height versus flux for an non-irradiated single-crystal sample (top left) a singlecrystal sample irradiated in the pilot run of the PLT (bottom). The average pulse height at the lowest flux is scaled to one, the average pulse heights at other fluxes were scaled by the same factor. The average cluster size in number of pixels versus flux for the non-irradiated single-crystal sample (top right).

\section{3D Detectors}

Another approach to increasing the charge yield of material is to use a so-called $3 D$ geometry to reduce the drift path by locating the electrodes in the bulk material. Recently results for a 
prototype single-crystal CVD diamond sensor using a 3D read-out geometry were presented [3]. A femto-second laser was used to create the 3D structure of electrodes in the $4.7 \times 4.7 \mathrm{~mm}$ large and $470 \mu \mathrm{m}$ thick sample. The femto-second laser allowed transforming the diamond lattice into a combination of diamond-like carbon, amorphous carbon and graphitic material - this combination of materials acted as electrodes. The cell size was $150 \times 150 \mu \mathrm{m}^{2}$ and each cell had a readout column at its centre and bias columns in the corners.

In addition to the 3D sensor two additional regions were created on the material: a planar strip detector with a strip pitch of $50 \mu \mathrm{m}$ and a 3D sensor without conductive channels. For measurements the planar strip was operated at a bias voltage of $500 \mathrm{~V}$ while the two 3D sensors were biased at $25 \mathrm{~V}$. This device was tested in a beam of $120 \mathrm{GeV}$ protons at the CERN-SPS. A silicon strip telescope was used to reconstruct tracks and extrapolate the intersection with the diamond device. The charge was then determined as the sum of charges from the three readout channels closest to the predicted intersection point for both the strip detector and 3D detector analyses.

An image of the raw data acquired can be seen in Fig.4 (left). Missing charge can be observed around the positions of broken readout columns whereas regions of small negative charge were found to be caused by broken field wires. As shown in Fig. 4 (right) very good agreement of the strip and 3D detector responses could be observed after correcting for these effects.
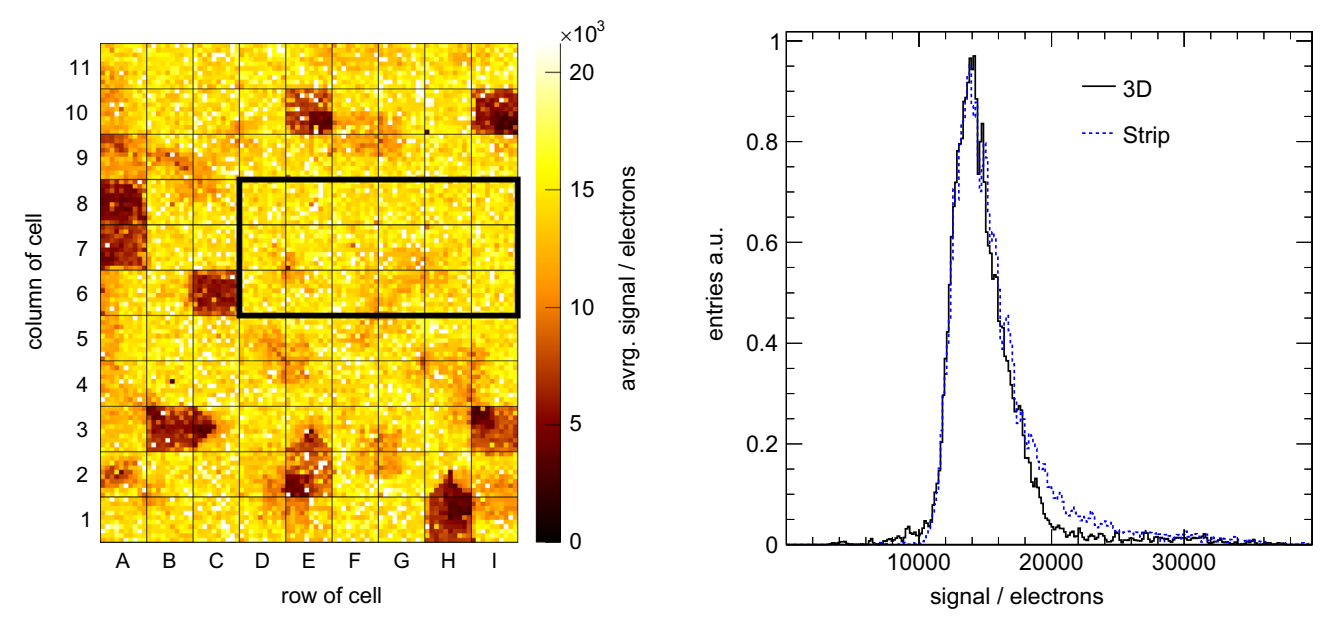

Figure 4: The average cluster charge versus predicted hit position (left). The fiducial region — chosen to contain adjacent cells with working readout columns — is highlighted. The transparent charge pulse height spectra of the strip detector and the 3D detector fiducial region (right) [3].

\section{Summary}

A flux dependence of the pulse height was previously observed in the pilot run of the PLT detector using a single-crystal CVD sensor operating the CASTOR region of CMS. The RD42 collaboration performed a dedicated beam test campaign to study the effects observed by the PLT. Irradiated pad and pixel diamond sensors were studied to disentangle geometric effects. The flux dependence could be replicated with single-crystal pixel sensors after neutron irradiation to a dose 
comparable to the total dose received by the PLT sensors. The effect is smaller for single-crystal pad sensors and was not observed for a poly-crystalline pad sensor up to particle flux of $300 \mathrm{kHz} / \mathrm{cm}^{2}$.

Progress has been made on a first 3D sensors using CVD diamonds as material. A first prototype is functional and shows encouraging results. Finally the ATLAS DBM using poly-crystalline CVD diamond sensors is currently observing first collisions in the LHC Run 2.

\section{References}

[1] E. Halkiadakis, et al. A Proposed Luminosity Monitor for CMS based on Small Angle Diamond Pixel Telescopes. Nucl. Instrum. Meth., A565(1):284-289, September 2006.

[2] A. Ferrari, P. R. Sala, A. Fasso, et al. FLUKA: A multi-particle transport code (Program version 2005). CERN-2005-010, SLAC-R-773, INFN-TC-05-11. , 2005.

[3] F. Bachmair et al. A 3D diamond detector for particle tracking. Nucl. Instrum. Meth., A786:97-104, 2015.

[4] D. Asner, et al. [RD42 Collaboration]. Diamond pixel modules. Nucl. Instrum. Meth., A636(1):S125-S129, April 2011.

[5] G. Battistoni, S. Muraro, P. R. Sala, et al. The FLUKA code: Description and benchmarking. AIP Conf. Proc., 896:31-49, 2007.

[6] H. Kagan, M. Mikuz,W. Trischuk. ATLAS Diamond Beam Monitor TDR. ATLAS Document DBM 001, 2011.

[7] H. Chr. Kästli, M. Barbero, W. Erdmann, Ch. Hormann, R. Horisberger, et al. Design and performance of the CMS pixel detector readout chip. Nucl. Instrum. Meth., A565:188-194, 2006.

[8] S. Müller. The beam condition monitor 2 and the radiation environment of the CMS detector at the LHC. PhD thesis, CERN-THESIS-2011-085, 2011.

[9] Ortec was taken over by PerkinElmer Instruments. 142A,C,B Preamplifiers. http://www.jlab.org/accel/inj_group/testcave/mott/142abc.htm.

[10] S. Ritt. DRS4 evaluation board. http://www.psi.ch/drs/evaluation-board.

[11] S. Schnetzer, et al. [RD42 Collaboration]. Diamond Sensors for Energy Frontier Experiments. Proceedings of Science, (Vertex 2013), 029, 2013.

[12] W. Adam, et al. [RD42 Collaboration]. Development of Diamond Tracking Detectors for High Luminosity Experiments at the LHC. Proposal/RD42 CERN/DRDC 94-21, Status Report/RD42, CERN/LHCC, 95-43, 95-53, 95-58, 97-03, 98-20, 2000-011, 2000-015, 2001-002, 2002-010, 2003-063, 2005-003, 2006-010, 2007-002, 2008-005.

[13] R. Wallny. Beam test results of the dependence of signal size on incident particle flux in diamond pixel and pad detectors. JINST, 10(07):C07009, 2015. 